\title{
Viscous dissipative effects in isotropic brane cosmology
}

\author{
Chiang-Mei Chen* \\ Department of Physics, National Taiwan University, Taipei 106, Taiwan \\ T. Harko ${ }^{\dagger}$ \\ Department of Physics, The University of Hong Kong, Pokfulam, Hong Kong
}

M. K. Mak

Department of Physics, The Hong Kong University of Science and Technology, Clear Water Bay, Hong Kong

(Received 2 July 2001; published 28 November 2001)

\begin{abstract}
We consider the dynamics of a viscous cosmological fluid in the generalized Randall-Sundrum model for an isotropic brane. To describe the dissipative effects we use the Israel-Hiscock-Stewart full causal thermodynamic theory. In the limiting case of a stiff cosmological fluid with pressure equal to the energy density, the general solution of the field equations can be obtained in an exact parametric form for a cosmological fluid with a constant bulk viscosity and with a bulk viscosity coefficient proportional to the square root of the energy density, respectively. The obtained solutions describe generally noninflationary brane worlds, starting from a singular state. During this phase of evolution the comoving entropy of the Universe is an increasing function of time, and thus a large amount of entropy is created in the brane world due to viscous dissipative processes.

DOI: 10.1103/PhysRevD.64.124017

PACS number(s): 04.20.Jb, 04.65.+e, 98.80.-k
\end{abstract}

\section{INTRODUCTION}

The idea $[1,2]$ that our four-dimensional Universe might be a three-brane embedded in a higher-dimensional space time has recently attracted much attention. According to the brane-world scenario, the physical fields in our fourdimensional space time, which are assumed to arise as fluctuations of branes in string theories, are confined to the three brane, while gravity can freely propagate in the bulk space time, with the gravitational self-couplings not significantly modified. This model arose from the study of a single 3 -brane embedded in five dimensions, with the 5D metric given by $d s^{2}=e^{-f(y)} \eta_{\mu \nu} d x^{\mu} d x^{\nu}+d y^{2}$, which can produce a large hierarchy between the scale of particle physics and gravity due to the appearance of the warp factor. Even if the fifth dimension is uncompactified, standard 4D gravity is reproduced on the brane. Hence this model allows the presence of large or even infinite noncompact extra dimensions and our brane is identified to a domain wall in a five-dimensional anti-de Sitter (AdS) space time.

The Randall-Sundrum model was inspired by superstring theory. The ten-dimensional $E_{8} \times E_{8}$ heterotic string theory, which contains the standard model of elementary particle, could be a promising candidate for a description of the real Universe. This theory is connected with an 11-dimensional theory, M theory, compactified on the orbifold $R^{10} \times S^{1} / Z_{2}$ [3]. In this model we have two separated ten-dimensional manifolds. For a recent review of the dynamics and geometry of brane universes see [4].

The static Randall-Sundrum solution has been extended to time-dependent solutions and their cosmological properties

\footnotetext{
*Email address: cmchen@phys.ntu.edu.tw

†Email address: tcharko@hkusua.hku.hk

‡Email address: mkmak@vtc.edu.hk
}

have been extensively studied [5-16]. In one of the first cosmological applications of this scenario it was pointed out that a model with a noncompact fifth dimension is potentially viable, while the scenario that might solve the hierarchy problem predicts a contracting Universe, leading to a variety of cosmological problems [17]. By adding cosmological constants to the brane and bulk, the problem of the correct behavior of the Hubble parameter on the brane has been solved by Cline et al. [18]. As a result one also obtains normal expansion during nucleosynthesis, but faster than normal expansion in the very early Universe. The creation of a spherically symmetric brane world in AdS bulk has been considered, from a quantum cosmological point of view, with the use of the Wheeler-de Witt equation, by Anchordoqui et al. [19].

The effective gravitational field equations on the brane world, in which all the matter forces except gravity are confined on the 3-brane in a five-dimensional space time with $Z_{2}$-symmetry, have been obtained by using an elegant geometric approach, by Shiromizu et al. [20,21]. The correct signature for gravity is provided by the brane with positive tension. If the bulk space time is exactly anti-de Sitter, generically the matter on the brane is required to be spatially homogeneous. The electric part of the five-dimensional Weyl tensor $E_{I J}$ gives the leading-order corrections to the conventional Einstein equations on the brane. The four-dimensional field equations for the induced metric and scalar field on the world volume of a 3-brane in the five-dimensional bulk with Einstein gravity plus a self-interacting scalar field have been derived by Maeda and Wands [22]. The effective fourdimensional Einstein equations include terms due to scalar fields and gravitational waves in the bulk.

The linearized perturbation equations in the generalized Randall-Sundrum model have been obtained, by using the covariant nonlinear dynamical equations for the gravitational and matter fields on the brane, by Maartens [23]. The nonlo- 
cal energy density determines the tidal acceleration in the off-brane direction and can oppose singularity formation via the generalized Raychaudhuri equation. Isotropy of the cosmic microwave background may no longer guarantee a Friedmann-Robertson-Walker (FRW) geometry. Vorticity on the brane decays as in general relativity, but nonlocal bulk effects can source the gravitomagnetic field, so that vector perturbations can also be generated in the absence of vorticity.

A systematic analysis, using dynamical systems techniques, of the qualitative behavior of the FRW, Bianchi type I and $\mathrm{V}$ cosmological models in the Randall-Sundrum braneworld scenario, with matter on the brane obeying a barotropic equation of state $p=(\gamma-1) \rho$, has been realized by Campos and Sopuerta [24,25]. In particular, they constructed the state spaces for these models and discussed what new critical points appear, the occurrence of bifurcations and the dynamics of the anisotropy for both a vanishing and nonvanishing Weyl tensor in the bulk.

All these investigations of brane cosmological models have been performed under the simplifying assumption of a perfect cosmological fluid. But in many cosmological situations an idealized fluid model of matter is inappropriate, especially in the case of matter at very high densities and pressures. Such possible situations are the relativistic transport of photons, mixtures of cosmic elementary particles, evolution of cosmic strings due to their interaction with each other and surrounding matter, classical description of the (quantum) particle production phase, interaction between matter and radiation, quark and gluon plasma viscosity, etc. From a physical point of view the inclusion of dissipative terms in the energy-momentum tensor of the cosmological fluid seems to be the best motivated generalization of the matter term of the gravitational field equations.

The first attempts at creating a theory of relativistic fluids were those of Eckart [26] and Landau and Lifshitz [27]. These theories are now known to be pathological in several respects. Regardless of the choice of equation of state, all equilibrium states in these theories are unstable and in addition signals may be propagated through the fluid at velocities exceeding the speed of light. These problems arise due to the first-order nature of the theory, that is, it considers only firstorder deviations from the equilibrium leading to parabolic differential equations, hence to infinite speeds of propagation for heat flow and viscosity, in contradiction with the principle of causality. Conventional theory is thus applicable only to phenomena that are quasistationary, i.e., slowly varying on space and time scales characterized by mean free path and mean collision time.

A relativistic second-order theory was found by Israel [28] and developed by Israel and Stewart [29] into what is called "transient" or "extended" irreversible thermodynamics. In this model deviations from equilibrium (bulk stress, heat flow, and shear stress) are treated as independent dynamical variables, leading to a total of 14 dynamical fluid variables to be determined. However, Hiscock and Lindblom [30] and Hiscock and Salmonson [31] have shown that most versions of the causal second-order theories omit certain divergence terms. The truncated causal thermodynamics of bulk viscosity leads to pathological behavior in the late Universe while the solutions of the full causal theory are [32] (a) for stable fluid configurations the dissipative signals propagate causally, (b) unlike in Eckart-type theories, there is no generic short-wavelength secular instability, and (c) even for rotating fluids, the perturbations have a well-posed initial value problem. For general reviews on causal thermodynamics and its role in relativity see [33] and [34].

Causal bulk viscous thermodynamics has been extensively used for describing the dynamics and evolution of the early Universe or in an astrophysical context. But due to the complicated character of the evolution equations, very few exact cosmological solutions of the gravitational field equations are known in the framework of the full causal theory. For a homogeneous Universe filled with a full causal viscous fluid source obeying the relation $\xi \sim \rho^{1 / 2}$, exact general solutions of the field equations have been obtained in [35-37]. In this case the evolution of the bulk viscous cosmological model can be reduced to a Painleve-Ince type differential equation, whose invariant form can be reduced, by means of nonlocal transformations, to a linear inhomogeneous ordinary second-order differential equation with constant coefficients [38]. It has also been proposed that causal bulk viscous thermodynamics can model on a phenomenological level matter creation in the early Universe [36,39]. Exact causal viscous cosmologies with $\xi \sim \rho^{s}$ have been obtained in [40].

Because of technical reasons, most investigations of dissipative causal cosmologies have assumed FRW symmetry (i.e., homogeneity and isotropy) or small perturbations around it [41]. The Einstein field equations for homogeneous models with dissipative fluids can be decoupled and therefore are reduced to an autonomous system of first-order ordinary differential equations, which can be analyzed qualitatively $[42,43]$.

It is the purpose of the present paper to investigate the effects of the bulk viscosity of the cosmological matter fluid on the dynamics of the brane world. Since the effects of the extra dimensions and also the viscous effects are more important at high matter densities, we restrict our analysis to the extreme case of a stiff (Zeldovich type) cosmological fluid, with pressure equal to the energy density. Hence the most important contribution to the energy density of the matter comes from the quadratic term in density, and during this period the energy density of matter is proportional to the Hubble parameter, in opposition to the standard general relativistic case with energy density proportional to the square of the Hubble parameter. In this case, and by assuming that the bulk viscosity coefficient and the temperature dependence of the cosmic fluid on the energy density are given by simple power laws, the field equations can be solved exactly for several explicit functional forms of the viscosity coefficient.

The present paper is organized as follows. The field equations on the brane describing the evolution of a viscous cosmological fluid are written down in Sec. II. In Sec. III we present the general solution of the field equations for a constant bulk viscosity and a bulk viscosity coefficient proportional to the square root of the energy density. The study of the stability of the equilibrium points of the dynamical sys- 
tem associated to the evolution of the viscous cosmological fluid is performed in Sec. IV. In Sec. V we discuss and conclude our results.

\section{DISSIPATIVE COSMOLOGICAL FLUIDS ON THE BRANE}

In the $5 \mathrm{D}$ space time the brane world is located as $Y\left(X^{I}\right)=0$, where $X^{I}, I=0,1,2,3,4$ are five-dimensional coordinates. The effective action in five dimensions is [22]

$$
\begin{aligned}
S= & \int d^{5} X \sqrt{-g_{5}}\left(\frac{1}{2 k_{5}^{2}} R_{5}-\Lambda_{5}\right) \\
& +\int_{Y=0} d^{4} x \sqrt{-g}\left(\frac{1}{k_{5}^{2}} K^{ \pm}-\lambda+L^{\text {matter }}\right),
\end{aligned}
$$

with $k_{5}^{2}=8 \pi G_{5}$ the five-dimensional gravitational coupling constant and where $x^{\mu}, \mu=0,1,2,3$ are the induced fourdimensional brane-world coordinates. $R_{5}$ is the $5 \mathrm{D}$ intrinsic curvature in the bulk and $K^{ \pm}$is the intrinsic curvature on either side of the brane.

On the five-dimensional space time (the bulk), with the negative vacuum energy $\Lambda_{5}$ and brane energy momentum as the source of the gravitational field, the Einstein field equations are given by

$$
G_{I J}=k_{5}^{2} T_{I J}, \quad T_{I J}=-\Lambda_{5} g_{I J}+\delta(Y)\left[-\lambda g_{I J}+T_{I J}^{\text {matter }}\right] .
$$

In this space time a brane is a fixed point of the $Z_{2}$ symmetry. In the following capital Latin indices run in the range $0, \ldots, 4$ while Greek indices take the values $0, \ldots, 3$.

Assuming a metric of the form $d s^{2}=\left(n_{I} n_{J}+g_{I J}\right) d x^{I} d x^{J}$, with $n_{I} d x^{I}=d \chi$ the unit normal to the $\chi=$ const hypersurfaces and $g_{I J}$ the induced metric on $\chi=$ const hypersurfaces, the effective four-dimensional gravitational equations on the brane (which are the consequence of the Gauss-Codazzi equations) take the form [20,21]:

$$
G_{\mu \nu}=-\Lambda g_{\mu \nu}+k_{4}^{2} T_{\mu \nu}+k_{5}^{4} S_{\mu \nu}-E_{\mu \nu},
$$

where $S_{\mu \nu}$ is the local quadratic energy-momentum correction

$$
S_{\mu \nu}=\frac{1}{12} T T_{\mu \nu}-\frac{1}{4} T_{\mu}{ }^{\alpha} T_{\nu \alpha}+\frac{1}{24} g_{\mu \nu}\left(3 T^{\alpha \beta} T_{\alpha \beta}-T^{2}\right),
$$

and $E_{\mu \nu}$ is the nonlocal effect from the bulk free gravitational field, transmitted projection of the bulk Weyl tensor $C_{I A J B}$,

$$
E_{I J}=C_{I A J B} n^{A} n^{B}, \quad E_{I J} \rightarrow E_{\mu \nu} \delta_{I}^{\mu} \delta_{J}^{\nu} \text { as } \chi \rightarrow 0 .
$$

The four-dimensional cosmological constant, $\Lambda$, and the coupling constant, $k_{4}$, are given by

$$
\Lambda=\frac{k_{5}^{2}}{2}\left(\Lambda_{5}+\frac{k_{5}^{2} \lambda^{2}}{6}\right), \quad k_{4}^{2}=\frac{k_{5}^{4} \lambda}{6} .
$$

The Einstein equation in the bulk, Codazzi equation, also implies the conservation of the energy-momentum tensor of the matter on the brane

$$
D_{\nu} T_{\mu}{ }^{\nu}=0 .
$$

Moreover, the contracted Bianchi identities on the brane imply that the projected Weyl tensor should obey the constraint

$$
D_{\nu} E_{\mu}{ }^{\nu}=k_{5}^{4} D_{\nu} S_{\mu}{ }^{\nu} .
$$

Finally, Eqs. (3), (7), and (8) give the complete set of field equations for the brane gravitational field.

For any matter fields (scalar field, perfect fluids, kinetic gases, dissipative fluids, etc.) the general form of the brane energy-momentum tensor can be covariantly given as

$$
T_{\mu \nu}=\rho u_{\mu} u_{\nu}+p h_{\mu \nu}+\pi_{\mu \nu}+2 q_{(\mu} u_{\nu)} .
$$

The decomposition is irreducible for any chosen fourvelocity $u^{\mu}$. Here $\rho$ and $p$ are the energy density and isotropic pressure, and $h_{\mu \nu}=g_{\mu \nu}+u_{\mu} u_{\nu}$ projects orthogonal to $u^{\mu}$. The energy flux obeys $q_{\mu}=q_{\langle\mu\rangle}$, and the anisotropic stress obeys $\pi_{\mu \nu}=\pi_{\langle\mu \nu\rangle}$, where angular brackets denote the projected, symmetric, and tracefree part:

$$
V_{\langle\mu\rangle}=h_{\mu}{ }^{\nu} V_{\nu}, \quad W_{\langle\mu \nu\rangle}=\left[h_{(\mu}{ }^{\alpha} h_{\nu)}{ }^{\beta}-\frac{1}{3} h^{\alpha \beta} h_{\mu \nu}\right] W_{\alpha \beta} .
$$

The symmetric properties of $E_{\mu \nu}$ imply that, in general, we can decompose it irreducibly with respect to a chosen four-velocity field $u^{\mu}$ as

$$
E_{\mu \nu}=-k^{4}\left[\mathcal{U}\left(u_{\mu} u_{\nu}+\frac{1}{3} h_{\mu \nu}\right)+\mathcal{P}_{\mu \nu}+2 \mathcal{Q}_{(\mu} u_{\nu)}\right],
$$

where $k=k_{5} / k_{4}$. In Eq. (11) $\mathcal{U}$ is the effective nonlocal energy density of the brane arising from the free gravitational field in the bulk, $\mathcal{P}_{\mu \nu}$ is the nonlocal anisotropic stress carrying Coulomb, gravitomagnetic, and gravitational wave effects from the bulk, while $\mathcal{Q}$ is the effective nonlocal energy flux on the brane.

The effect of the bulk viscosity of the cosmological fluid can be considered by adding to the usual thermodynamic pressure $p$ the bulk viscous pressure $\Pi$ and formally substituting the pressure terms in the energy-momentum tensor by $p_{\text {eff }}=p+\Pi$. The particle flow vector $N^{\mu}$ is given by $N^{\mu}$ $=n u^{\mu}$, where $n \geqslant 0$ is the particle number density.

In the framework of causal thermodynamics, and limiting ourselves to second-order deviations from equilibrium, the entropy flow vector $S^{\mu}$ takes the form

$$
S^{\mu}=s N^{\mu}-\frac{\tau \Pi^{2}}{2 \xi T} u^{\mu}
$$

where $s$ is the entropy per particle, $\tau$ the relaxation time, $T$ the temperature, and $\xi$ is the bulk viscosity coefficient. 
We consider that the heat transfer is zero, $q_{\mu}=0$ in Eq. (9), and also a vanishing effective nonlocal anisotropic stress and energy flux $\mathcal{P}_{\mu \nu}=0=\mathcal{Q}_{\mu}$. Then the matter corrections are given by

$$
S_{\mu \nu}=\frac{1}{12} \rho^{2} u_{\mu} u_{\nu}+\frac{1}{12} \rho\left(\rho+2 p_{\text {eff }}\right) h_{\mu \nu} .
$$

The line element of a flat Robertson-Walker metric is given by

$$
d s^{2}=-d t^{2}+a^{2}(t)\left(d x^{2}+d y^{2}+d z^{2}\right) .
$$

We also assume that the thermodynamic pressure $p$ of the cosmological fluid obeys a linear barotropic equation of state $p=(\gamma-1) \rho, \gamma=$ const and $1 \leqslant \gamma \leqslant 2$. Under these assumptions, the field equations and the conservation equations for the Bianchi type I brane gravitational field take the form

$$
\begin{aligned}
3 H^{2}= & \Lambda+k_{4}^{2} \rho+\frac{k_{4}^{2}}{2 \lambda} \rho^{2}+\frac{6 \mathcal{U}}{k_{4}^{2} \lambda}, \\
2 \dot{H}+3 H^{2}= & \Lambda-k_{4}^{2}[(\gamma-1) \rho+\Pi] \\
& -\frac{k_{4}^{2}}{2 \lambda}\left[(2 \gamma-1) \rho^{2}+2 \rho \Pi\right]-\frac{2 \mathcal{U}}{k_{4}^{2} \lambda}, \\
\dot{\rho}+3 \gamma H \rho= & -3 H \Pi, \\
\dot{\mathcal{U}}+4 H \mathcal{U}= & 0,
\end{aligned}
$$

where the Hubble parameter $H$ is defined as $H=\dot{a} / a . N^{\mu}{ }_{; \mu}$ $=0$ leads to the particle number conservation equation $\dot{n}$ $+3 H n=0$.

The causal evolution equation for the bulk viscous pressure $\Pi$ is given by [33]

$$
\tau \Pi+\Pi=-3 \xi H-\frac{1}{2} \tau \Pi\left(3 H+\frac{\dot{\tau}}{\tau}-\frac{\dot{\xi}}{\xi}-\frac{\dot{T}}{T}\right) .
$$

Equation (19) arises as the simplest way (linear in $\Pi$ ) to satisfy the $H$ theorem [i.e., for the entropy production to be nonnegative, $\left.S_{; \mu}^{\mu}=\Pi^{2} /(\xi T) \geqslant 0\right]$. The Israel-Stewart theory is derived under the assumption that the thermodynamic state of the fluid is close to equilibrium, which means that the nonequilibrium bulk viscous pressure should be small when compared to the local equilibrium pressure, that is $|\Pi|<p$.

The growth of the total comoving entropy $\Sigma(t)$ over a proper time interval $\left(t_{0}, t\right)$ is given by [34]

$$
\Sigma(t)-\Sigma\left(t_{0}\right)=-\frac{3}{k_{B}} \int_{t_{0}}^{t} \frac{\prod a^{3} H}{T} d t .
$$

An important observational quantity is the deceleration parameter $q=d H^{-1} / d t-1$. The sign of the deceleration parameter indicates whether the model inflates or not. The positive sign of $q$ corresponds to "standard" decelerating models whereas the negative sign indicates inflation.
Since the effects of the extra dimensions are important at very high densities, when the cosmological fluid behaves like a Zeldovich fluid with $p=\rho(\gamma=2)$, as are also the dissipative effects, we consider only the physical situation in which the quadratic term dominates in the energy equation (15). Therefore during the early period of evolution the energy density of the Universe is given approximately by $\rho$ $\approx 3 \rho_{0} H$, with $3 \rho_{0}=\sqrt{6 \lambda / k_{4}^{2}}$.

In order to close the system of Eqs. (15)-(19) we have to specify $T, \tau$, and $\xi$.

First, following [44] we suppose that the relation $\tau=\xi / \rho$ holds in order to guarantee that the propagation velocity of bulk viscous perturbations, i.e., the nonadiabatic contribution to the speed of sound in a dissipative fluid without heat flux or shear viscosity, does not exceed the speed of light. An analysis of the relativistic kinetic equation for some simple cases given by Belinskii and Khalatnikov [45], Belinskii et al. [44] and Murphy [46] has shown that in the asymptotic regions of small and large values of the energy density, the viscosity coefficients can be approximated by power functions of the energy density with definite requirements on the exponents of these functions. For small values of the energy density it is reasonable to consider large exponents, equal in the extreme case to one. For large $\rho$ the power of the bulk viscosity coefficient should be considered smaller (or equal) to $1 / 2$.

Therefore we assume the following simple phenomenological laws for the bulk viscosity coefficient, temperature, and relaxation time:

$$
\xi=\alpha \rho^{s}=\xi_{0} H^{s}, \quad T=\beta \rho^{r}=T_{0} H^{r}, \quad \tau=\frac{\xi}{\rho}=\frac{H^{s-1}}{\tau_{0}},
$$

where $s \geqslant 0, r \geqslant 0, \alpha \geqslant 0$, and $\beta \geqslant 0$ are constants and $\xi_{0}$ $=\alpha\left(3 \rho_{0}\right)^{s}$ and $\tau_{0}=\xi_{0}^{-1}$.

In the context of irreversible thermodynamics, $p, \rho, T$ and the number density $n$ are equilibrium magnitudes which are generally related by equations of state of the form $\rho$ $=\rho(T, n)$ and $p=p(T, n)$. From the requirement that the entropy is a state function we obtain the equation

$$
\left(\frac{\partial \rho}{\partial n}\right)_{T}=\frac{\rho+p}{n}-\frac{T}{n}\left(\frac{\partial p}{\partial T}\right)_{n}
$$

which imposes the constraint $r=(\gamma-1) / \gamma$. Hence for a Zeldovich fluid we have $r=1 / 2$.

With these assumptions the bulk viscous pressure $\Pi$ can be obtained from Eq. (17) in the form

$$
\Pi=-\rho_{0}\left(\frac{\dot{H}}{H}+6 H\right) .
$$

The bulk viscous evolution equation (19) can be written as

$$
\Pi+\frac{1}{\tau} \Pi=-3 \rho H-\frac{1}{2} \Pi\left(3 H-\frac{3}{2} \frac{\dot{\rho}}{\rho}\right),
$$


and for a stiff cosmological fluid on the brane takes the form

$$
\frac{\ddot{H}}{H}-\frac{7}{4} \frac{\dot{H}^{2}}{H^{2}}+\left(3+\tau_{0} H^{-s}\right) \dot{H}+6 \tau_{0} H^{2-s}=0 .
$$

By means of the substitution $H=y^{-4 / 3}$, Eq. (25) takes the form

$$
\ddot{y}+\left(3+\tau_{0} y^{4 s / 3}\right) y^{-4 / 3} \dot{y}-\frac{9}{2} \tau_{0} y^{(4 s-5) / 3}=0 .
$$

By taking $u=\dot{y}$ and denoting $v=1 / u$, Eq. (26) can be transformed to a second type Abel nonlinear first-order differential equation:

$$
\frac{d v}{d y}-\left(3+\tau_{0} y^{4 s / 3}\right) y^{-4 / 3} v^{2}+\frac{9}{2} \tau_{0} y^{(4 s-5) / 3} v^{3}=0
$$

By introducing a new variable $\eta=3 y^{-1 / 3}$, Eq. (27) becomes

$$
\frac{d v}{d \eta}+\left(3+3^{4 s} \tau_{0} \eta^{-4 s}\right) v^{2}-\frac{3^{4 s+1}}{2} \tau_{0} \eta^{1-4 s} v^{3}=0
$$

\section{BRANE EVOLUTION OF DISSIPATIVE STIFF COSMOLOGICAL FLUIDS}

In the previous section we have formulated the basic equations describing the dynamics of a dissipative stiff cosmological fluid on the brane. We have considered only the extreme case of very high densities, when the main contribution to the energy of the matter is given by the quadratic term in the energy-momentum tensor, due to the form of the Gauss-Codazzi equations, and which leads to major changes in the dynamics of the Universe. In this case the basic equation describing the evolution of the Universe can be reduced to an Abel type equation (28).

It is the purpose of the present section to consider some exact classes of solutions of Eq. (28), corresponding to some particular values of the constant $s$.

As a first case we assume that the bulk viscosity coefficient $\xi$ is a constant, $\xi=\xi_{0}=$ const, corresponding to the choice $s=0$ in the equation of state of the bulk viscosity coefficient. For $s=0$ the temperature and the relaxation time are functions of density, according to the equations of state (21).

For $s=0$ the evolution equation (28) of the bulk viscous pressure takes the form

$$
\frac{d v}{d \eta}+\left(3+\tau_{0}\right) v^{2}-\frac{3}{2} \tau_{0} \eta v^{3}=0
$$

By introducing a new variable $\eta^{\prime}=\left(3+\tau_{0}\right) \eta$ and denoting $b=3 \tau_{0} / 2\left(3+\tau_{0}\right)^{2}$, Eq. (29) takes the form

$$
\frac{d v}{d \eta^{\prime}}+v^{2}-b \eta^{\prime} v^{3}=0
$$

By taking $v=w / \eta^{\prime}$, Eq. (30) is transformed into

$$
\frac{d w}{d \eta^{\prime}}=\frac{w}{\eta^{\prime}}\left(b w^{2}-w+1\right) .
$$

Hence the general solution of Eq. (30) is given by

$$
\eta^{\prime}=C \frac{w}{\sqrt{b w^{2}-w+1}} e^{f(w)}
$$

where $C>0$ is an arbitrary constant of integration,

$$
f(w)=\frac{1}{2} \int \frac{d w}{b w^{2}-w+1},
$$

and

$$
\begin{aligned}
& f(w)=\frac{1}{2 \sqrt{\Delta}} \ln \left(\frac{2 b w-1-\sqrt{\Delta}}{2 b w-1+\sqrt{\Delta}}\right), \quad \text { if } b<\frac{1}{4}, \\
& f(w)=-\frac{2}{w-2}, \quad \text { if } b=\frac{1}{4}, \\
& f(w)=\frac{1}{\sqrt{-\Delta}} \arctan \left(\frac{2 b w-1}{\sqrt{-\Delta}}\right), \quad \text { if } b>\frac{1}{4},
\end{aligned}
$$

where we denoted $\Delta=1-4 b$.

Therefore the general solution of the field equations can be expressed in the following exact parametric form, with $\theta=1 / w$ taken as parameter:

$$
\begin{aligned}
t(\theta)-t_{0} & =C_{0} \int\left(\theta^{2}-\theta+b\right) e^{-4 f(\theta)} d \theta \\
H(\theta) & =H_{0} \frac{e^{4 f(\theta)}}{\left(\theta^{2}-\theta+b\right)^{2}}, \\
a(\theta)= & a_{0} \exp \left[-2 C_{0} H_{0} f(\theta)\right] \\
q(\theta)= & 4 \theta / C_{0} H_{0}-1 \\
\Pi(\theta)= & -2 \rho_{0} \frac{e^{4 f(\theta)}}{\left(\theta^{2}-\theta+b\right)^{2}}\left(3 H_{0}-2 \theta / C_{0}\right) \\
\Sigma(\theta)= & \Sigma\left(\theta_{0}\right)+\frac{6 a_{0}^{3} \rho_{0} \sqrt{H_{0}}}{k_{B} T_{0}} \\
& \times \int \frac{\left(3 C_{0} H_{0}-2 \theta\right) \exp \left[2 f(\theta)\left(1-3 C_{0} H_{0}\right)\right]}{\left(\theta^{2}-\theta+b\right)^{2}} d \theta
\end{aligned}
$$

$$
\mathcal{U}(\theta)=\mathcal{U}_{0} \exp \left[8 C_{0} H_{0} f(\theta)\right]
$$

where $H_{0}=\left[C / 3\left(3+\tau_{0}\right)\right]^{4}, C_{0}=3^{4}\left(3+\tau_{0}\right)^{3} / C^{4}$, and $t_{0}, a_{0}$, and $\mathcal{U}_{0}$ are constants of integration. In the new variable $\theta$ the function $f$ is given by $f(\theta)=-2^{-1} \int\left(\theta^{2}-\theta+b\right)^{-1} d \theta$. 
The thermodynamic consistency of the model can be studied from the ratio of the bulk viscous and thermodynamic pressure, which is given by

$$
l=\left|\frac{\Pi}{p}\right|=\frac{1}{3}|5-q| .
$$

The second case we analyze corresponds to the extreme limit of very high densities when $s=1 / 2$. Then Eq. (28) takes the form

$$
\frac{d v}{d \eta}+\left(3+9 \tau_{0} \eta^{-2}\right) v^{2}-\frac{27}{2} \tau_{0} \eta^{-1} v^{3}=0
$$

Introducing two new functions $A(\eta)=-2 \eta^{2} / 9 \tau_{0}+2 / 3$ and $B(\eta)=-2 \eta / 27 \tau_{0}$ allows us to rewrite Eq. (45) in the general form

$$
\frac{d v}{d \eta}=-\frac{v^{3}}{B(\eta)}-\left[\frac{d}{d \eta} \frac{A(\eta)}{B(\eta)}\right] v^{2} .
$$

By introducing a new variable

$$
\sigma=\frac{1}{v}-\frac{A(\eta)}{B(\eta)}
$$

Eq. (46) can be written in the general form

$$
\frac{d \eta}{d \sigma}=B(\eta) \sigma+A(\eta)
$$

or, equivalently,

$$
\frac{d \eta}{d \sigma}=-\frac{2}{9 \tau_{0}} \eta^{2}-\frac{2}{27 \tau_{0}} \sigma \eta+\frac{2}{3} .
$$

Hence we have transformed the initial Abel type equation into a Riccati equation. A particular solution of Eq. (49) is given by

$$
\eta=9 \tau_{0} \sigma \Delta(\sigma)
$$

and therefore the general solution of Eq. (49) is

$$
\eta(\sigma)=9 \tau_{0} \sigma \Delta(\sigma)+\frac{\Delta^{2}(\sigma) e^{-\sigma^{2} / 27 \tau_{0}}}{C_{1}+\frac{2}{9 \tau_{0}} \int \Delta^{2}(\sigma) e^{-\sigma^{2} / 27 \tau_{0}} d \sigma},
$$

where $\Delta(\sigma)=\left(\sigma^{2}+27 \tau_{0} / 2\right)^{-1}$ and $C_{1}$ is a constant of integration.

Hence we obtain the general solution of the gravitational field equations on the brane for a Zeldovich causal bulk viscous fluid, with bulk viscosity coefficient proportional to the square root of the density, in the following exact parametric form, with $\sigma$ taken as parameter:

$t(\sigma)-t_{0}=6 \tau_{0}^{-1} \int \eta^{-3}(\sigma) d \sigma$

$$
\begin{aligned}
& H(\sigma)=\frac{1}{81} \eta^{4}(\sigma), \\
& a(\sigma)=a_{0} \exp \left[\int \frac{2 \eta(\sigma)}{27 \tau_{0}} d \sigma\right], \\
& q(\sigma)=\frac{4 \sigma}{\eta(\sigma)}-\frac{36 \tau_{0}}{\eta^{2}(\sigma)}+11, \\
& \Pi(\sigma)=\frac{2 \rho_{0}}{81} \eta^{2}(\sigma)\left[3 \eta^{2}(\sigma)+2 \sigma \eta(\sigma)-18 \tau_{0}\right],
\end{aligned}
$$

$$
\begin{aligned}
\Sigma(\sigma)= & \Sigma\left(\sigma_{0}\right)-\frac{4 \rho_{0} a_{0}^{3}}{81 \tau_{0} T_{0} k_{B}} \int \eta(\sigma)\left[3 \eta^{2}(\sigma)\right. \\
& \left.+2 \sigma \eta(\sigma)-18 \tau_{0}\right] \exp \left[\int \frac{2 \eta(\sigma)}{9 \tau_{0}} d \sigma\right] d \sigma
\end{aligned}
$$

$$
\mathcal{U}(\sigma)=\mathcal{U}_{0} \exp \left[-\frac{8}{27 \tau_{0}} \int \eta(\sigma) d \sigma\right]
$$

\section{STABILITY ANALYSIS OF THE EQUILIBRIUM POINTS OF THE VISCOUS COSMOLOGICAL FLUID}

The general evolution equation of the bulk viscous cosmological fluid on the brane is given by Eq. (25). From a mathematical point of view it is a second-order nonlinear differential equation of the form $\ddot{H}+R(H, \dot{H})=0$, with $R(H, \dot{H})=-7 \dot{H}^{2} / 4 H+\left(3 H+\tau_{0} H^{1-s}\right) \dot{H}+6 \tau_{0} H^{3-s}$. Therefore $R(0,0)=\lim _{H, X \rightarrow 0} R(H, X)=0$ and $R(H, 0) \neq 0$ for $H$ $\neq 0$.

In order to study the stability of the equilibrium points of the evolution equation of the viscous cosmological fluid on the brane, Eq. (25), we shall rewrite it in the form of an autonomous dynamical system, by introducing a new variable $X=\dot{H}$ :

$$
\begin{aligned}
& \frac{d H}{d t}=X, \\
& \frac{d X}{d t}=\frac{7 X^{2}}{4 H}-\left(3 H+\tau_{0} H^{1-s}\right) X-6 \tau_{0} H^{3-s} .
\end{aligned}
$$

The critical points of this dynamical system are given by $H=X=0$. They correspond to a Minkowskian space time $(a=$ const $=1)$ and to a de Sitter inflationary phase, with $a$ $=\exp \left(H_{0} t\right)$ and $H_{0}=$ const. The system has no other critical points besides the origin.

The Lyapunov function $V(H, X)$ associated to this system can be chosen [47] as $V(H, X)=X^{2} / 2+\int_{0}^{H} R(s, 0) d s$ and is given by

$$
V(H, X)=\frac{1}{2} X^{2}+\frac{6 \tau_{0}}{4-s} H^{4-s}
$$




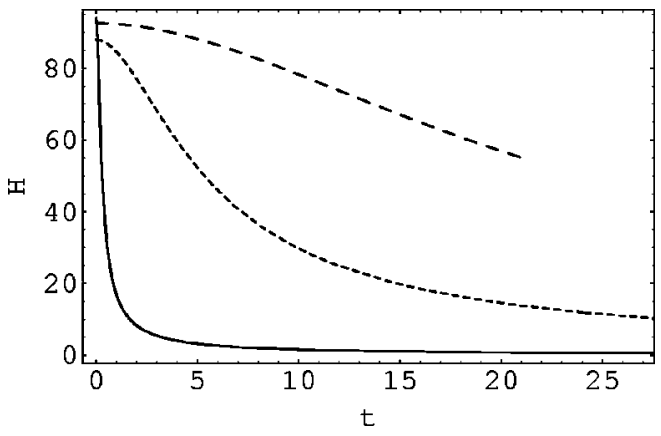

FIG. 1. Variation as a function of time of the Hubble parameter $H$ of the brane Universe with confined dissipative cosmological fluid with constant coefficient of bulk viscosity for different values of the parameter $b: b=1 / 6$ (solid curve), $b=1 / 4$ (dotted curve), and $b=1 / 2$ (dashed curve). The values of the constants $H_{0}, C_{0}$, and $t_{0}$ are different for each curve.

The Lyapunov function (61) has the properties $V(0,0)=0$ and $d V / d t=7 X^{3} / 4 H-\left(3 H+\tau_{0} H^{1-s}\right) X^{2}$. According to the standard theory of this type of differential equation [47] the equilibrium state $(H=0, X=0)$ is stable if the conditions

$$
H R(H, 0)=6 \tau_{0} H^{4-s}>0, \quad \text { for } H \neq 0,
$$

and

$$
\begin{aligned}
\frac{\partial R(H, X)}{\partial X} & =-\frac{7 X}{2 H}+3 H+\tau_{0} H^{1-s} \\
& =H\left[\frac{7}{2}(q+1)+3+\tau_{0} H^{-s}\right] \geqslant 0,
\end{aligned}
$$

holds, where the deceleration parameter $q=-X / H^{2}-1$. Moreover, if the condition $\partial R(H, X) / \partial X>0$ is satisfied for $H X \neq 0$, the equilibrium state is asymptotically stable [47]. The equilibrium state is unstable if $\partial R(H, X) / \partial X<0$ for $H X \neq 0$.

The stability criteria of the critical point can be formulated in terms of some conditions imposed on the deceleration parameter. In the limit of large time, $H \rightarrow 0$ and the term $H^{-s}>0$ dominates in the expression of $\partial R(H, X) / \partial X$, making it obviously non-negative. In the small time limit, $H$ $\rightarrow \infty$ and the condition of the stability of the critical point is $7(q+1) / 2+3 \geqslant 0$, or $q \geqslant-13 / 7$. If $q>-13 / 7$ the critical point is also asymptotically stable. On the other hand for $q$ $<-13 / 7$ the equilibrium point is unstable.

\section{DISCUSSIONS AND FINAL REMARKS}

In the present paper we have considered the evolution of a causal viscous dissipative cosmological fluid in the braneworld scenario. As the only source of dissipation we have considered the bulk viscosity of the matter on the brane. The most important differences to standard general relativity are expected to occur in the limit of extremely high densities, when the fluid obeys a Zeldovich (stiff) equation of state $\rho$ $=p$. In this case the Friedmann equation is modified due to the presence of the terms from extra dimensions, quadratic in the energy density, which dominates the other terms in the

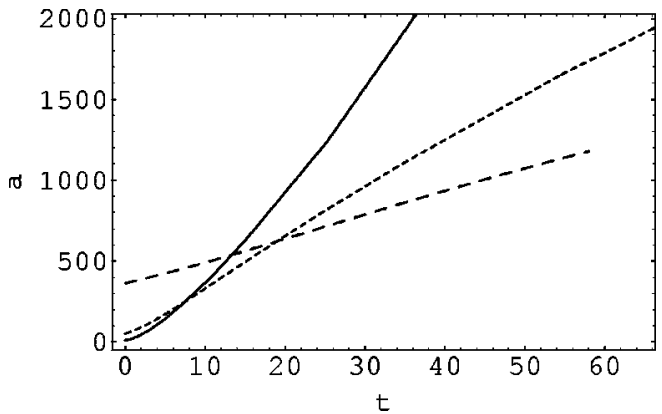

FIG. 2. Time evolution of the scale factor $a$ of the brane Universe with confined dissipative cosmological fluid with constant coefficient of bulk viscosity for different values of the parameter $b$ : $b=1 / 6$ (solid curve), $b=1 / 4$ (dotted curve), and $b=1 / 2$ (dashed curve). The values of the constants $a_{0}, C_{0}$, and $t_{0}$ are different for each curve.

energy-momentum tensor, leading to an energy density of the cosmic fluid proportional to the Hubble parameter.

By assuming the usual equations of state for bulk viscosity, temperature, and relaxation time, the field equations can be solved exactly for some specific choices of the constant $s$ describing the bulk viscosity coefficient-energy density functional relation.

For the $s=0$ case corresponding to a constant bulk viscosity coefficient $\xi=$ const, the general solution of the field equations for the viscous fluid on the brane world is given by Eqs. (37)-(43). Since the bulk viscous pressure $\Pi$ must be negative, $\Pi \leqslant 0$, it follows that in order to satisfy this condition the range of the parameter $\theta$ must be restricted to $\theta$ $\in[0,2 / 3]$.

In the limit of small times, we have $\theta \rightarrow 0$ and one obtains the following equations describing the evolution of the viscous cosmological fluid on the brane:

$$
\begin{aligned}
& a \sim t^{C_{0} H_{0} / 2}, \quad H \sim t^{-2 C_{0} H_{0} t}, \quad \rho=p \sim t^{-2 C_{0} H_{0} t}, \quad q=-1, \\
& \Pi \sim-t^{-1}, \quad \mathcal{U} \sim t^{-2 C_{0} H_{0}}, \quad \Sigma(t) \sim \Sigma\left(t_{0}\right)+t^{3\left(1-C_{0} H_{0}\right) / 2} .
\end{aligned}
$$

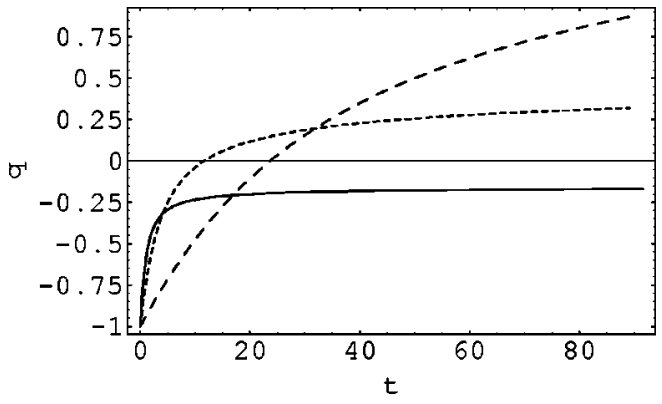

FIG. 3. Dynamics of the deceleration parameter $q$ of the brane Universe with confined dissipative cosmological fluid with constant coefficient of bulk viscosity for different values of the parameter $b$ : $b=1 / 6$ (solid curve), $b=1 / 4$ (dotted curve), and $b=1 / 2$ (dashed curve). The value of the constant $t_{0}$ is different for each curve and the constants $C_{0}$ and $H_{0}$ have been normalized so that $H_{0} C_{0}=1$. 


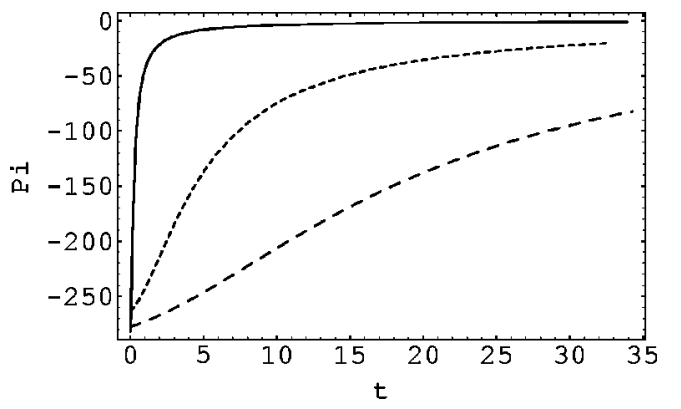

FIG. 4. Variation as a function of time of the bulk viscous pressure $\Pi$ of the brane Universe with confined dissipative cosmological fluid with constant coefficient of bulk viscosity for different values of the parameter $b: b=1 / 6$ (solid curve), $b=1 / 4$ (dotted curve), and $b=1 / 2$ (dashed curve). The values of the constants $H_{0}$, $C_{0}$, and $t_{0}$ are different for each curve.

The variations of the scale factor, Hubble parameter, deceleration parameter, bulk viscous pressure, and comoving entropy of the constant bulk viscosity coefficient dissipative cosmological fluid confined on the brane are presented in Figs. 1-5.

The evolution of the viscous brane Universe starts from a singular state, with infinite values of the energy density and pressure and zero scale factor. The initial evolution is inflationary, with a negative deceleration parameter. But in the large-time limit the dynamics becomes noninflationary, with the deceleration parameter $q>0$. Therefore the inclusion of viscous effects during the period when the quadratic term in energy density (coming from extra dimensions) dominates the dynamics of the space time, provides an effective mechanism for the "graceful exit" of the brane world from the initial inflationary phase to a noninflationary era. Because of dissipative effects the entropy on the brane is increasing in time and a large amount of entropy is produced in the early stages of the evolution of the brane Universe. The nonlocal energy density on the brane, $\mathcal{U}$, is a decreasing function of time, so the effects of the gravitational field on the bulk become rapidly negligible. But the criterion of the thermodynamic consistency of the model, $l=|\Pi / p|<1$, is not generally satisfied in this model, as can be easily seen from Eq.

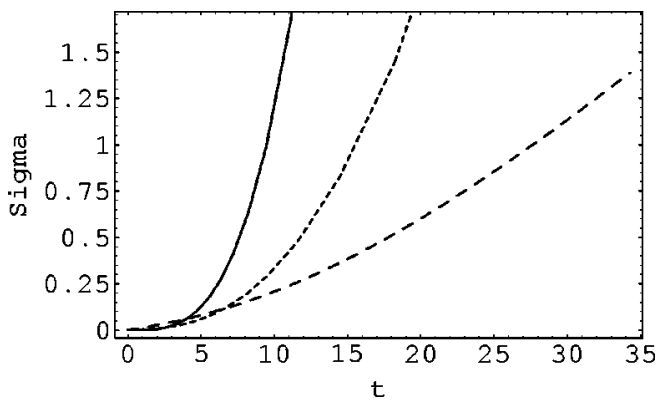

FIG. 5. Time evolution of the comoving entropy $\Sigma$ of the brane Universe with confined dissipative cosmological fluid with constant coefficient of bulk viscosity for different values of the parameter $b$ : $b=1 / 6$ (solid curve), $b=1 / 4$ (dotted curve), and $b=1 / 2$ (dashed curve). The values of the constants $H_{0}, C_{0}, t_{0}$, and $a_{0}$ are different for each curve.

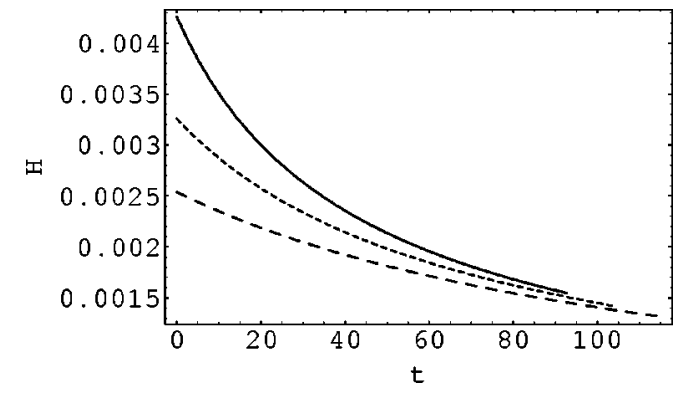

FIG. 6. Variation as a function of time of the Hubble parameter $H$ of the brane Universe with confined dissipative cosmological fluid with the coefficient of bulk viscosity proportional to the square root of the energy density $(s=1 / 2)$ for different numerical values of the integration constant $C_{1}: C_{1}=0.58$ (solid curve), $C_{1}=0.62$ (dotted curve), and $C_{1}=0.66$ (dashed curve) $\left(t_{0}=0\right)$. The constant $\tau_{0}$ has been normalized so that $\tau_{0}=1$.

(44). All the inflationary states clearly contradict the condition. On the other hand it is possible to find some particular of the parameter $b$ leading to thermodynamic consistency during the noninflationary phase.

The time variations of the Hubble parameter, scale factor, deceleration parameter, bulk viscous pressure, and comoving entropy for the brane Universe with a dissipative cosmological fluid with the bulk viscosity coefficient proportional to the square root of the energy density $(s=1 / 2)$ are represented in Figs. 6-10.

The behavior of the Universe depends on the numerical values of the arbitrary integration constant $C_{1}$ and of the constant $\tau_{0}$. For the chosen numerical values of these parameters the Universe generally starts from a singular state, with zero and infinite values of the scale factor and energy density, respectively. In order that the model represents a dissipative fluid, with negative bulk viscous pressure, the parameter $\sigma$ must satisfy the condition $2 \sigma<18 \tau_{0} / \eta(\sigma)-3 \eta(\sigma)$. In opposition to the constant bulk viscosity case, the evolution is noninflationary for all times. Because of viscous dissipative effects, a large amount of comoving entropy is created on the brane and the entropy of the Universe is increasing due to viscous dissipation.

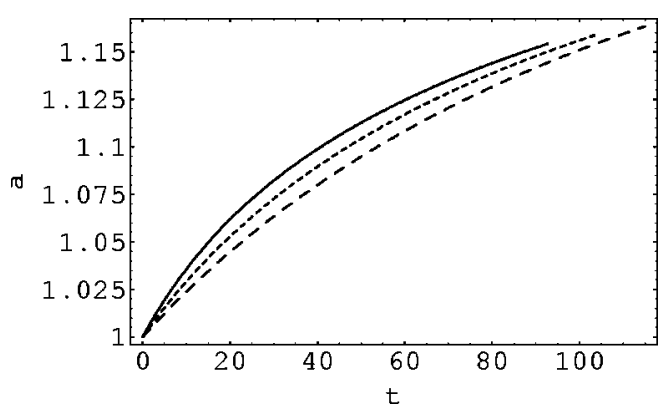

FIG. 7. Time evolution of the scale factor $a$ of the brane Universe with confined dissipative cosmological fluid with the coefficient of bulk viscosity proportional to the square root of the energy density $(s=1 / 2)$ for different numerical values of the integration constant $C_{1}: C_{1}=0.58$ (solid curve), $C_{1}=0.62$ (dotted curve), and $C_{1}=0.66$ (dashed curve) $\left(t_{0}=0\right)$. The constant $\tau_{0}$ has been normalized so that $\tau_{0}=1$. 


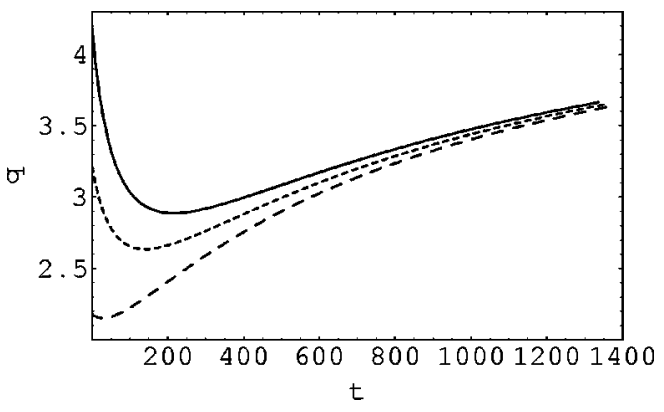

FIG. 8. Dynamics of the deceleration parameter $q$ of the brane Universe with confined dissipative cosmological fluid with the coefficient of bulk viscosity proportional to the square root of the energy density $(s=1 / 2)$ for different numerical values of the integration constant $C_{1}: C_{1}=0.58$ (solid curve), $C_{1}=0.62$ (dotted curve), and $C_{1}=0.66$ (dashed curve) $\left(t_{0}=0\right)$. The constant $\tau_{0}$ has been normalized so that $\tau_{0}=1$. The deceleration parameter satisfies the condition $q>0$ for all times.

Figure 11 presents the time variation of the ratio $l$ of the bulk viscous and thermodynamic pressures, respectively. For all times $|\Pi / p|<1$ and hence in this model the thermodynamical consistency condition of the smallness of the bulk viscous pressure is satisfied for all times and for a large class of admissible values of the integration constant $C_{1}$ and of $\tau_{0}$.

The effective nonlocal energy on the brane, $\mathcal{U}$, is tending rapidly to zero in the large time limit.

The general exact solution of the gravitational field equations for a homogeneous flat FRW Universe filled with a causal bulk viscous fluid with the bulk viscosity coefficient proportional to the Hubble function $\xi \sim \rho^{1 / 2} \sim H$ has been obtained, in the framework of the standard general relativity (GR), in [37]. The solution of the field equations can in this case also be represented in an exact parametric form. There are major differences between the general evolution of the dissipative cosmological fluids in the brane and GR models. In GR the dynamics of the cosmological fluid described by the general solution is inflationary for all times, with the thermodynamic consistency condition $|\Pi / p|<1$ violated

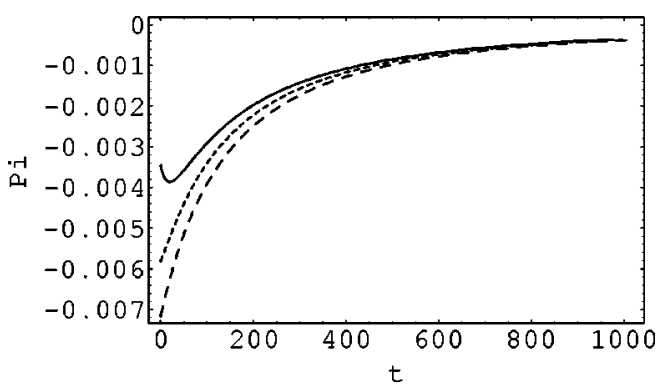

FIG. 9. Variation as a function of time of the bulk viscous pressure $\Pi$ of the brane Universe with confined dissipative cosmological fluid with the coefficient of bulk viscosity proportional to the square root of the energy density $(s=1 / 2)$ for different numerical values of the integration constant $C_{1}: C_{1}=0.58$ (solid curve), $C_{1}$ $=0.62$ (dotted curve), and $C_{1}=0.66$ (dashed curve) $\left(t_{0}=0\right)$. The constant $\tau_{0}$ has been normalized so that $\tau_{0}=1$. As required by the model, the bulk viscous pressure is negative for all times.

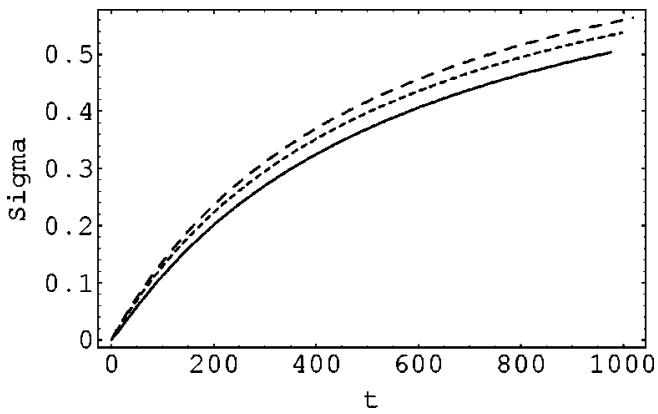

FIG. 10. Dynamics of the comoving entropy $\Sigma$ of the brane Universe with confined dissipative cosmological fluid with the coefficient of bulk viscosity proportional to the square root of the energy density $(s=1 / 2)$ for different numerical values of the integration constant $C_{1}: C_{1}=0.58$ (solid curve), $C_{1}=0.62$ (dotted curve), and $C_{1}=0.66$ (dashed curve) $\left(t_{0}=0\right)$. The constant $\tau_{0}$ has been normalized so that $\tau_{0}=1$.

during the entire expansionary evolution period. But in the brane model the evolution is noninflationary, with the consistency condition satisfied, at least for a specific range of values of the parameters $\tau_{0}$ and $C_{1}$, which are unknown for a realistic physical situation (of course for some particular numerical values of these parameters, inflationary initial behavior or increasing energy density can also be obtained). During the general relativistic inflationary period the density, temperature, bulk viscosity coefficient, and comoving entropy are rapidly increasing functions of time. In fact the general solution of the GR field equations describe a transition between two Minkowskian space times connected by an inflationary period. For some particular values of the parameters one can also obtain general relativistic noninflationary solutions [37].

The consideration of viscous dissipative effects in the brane and general relativistic models in the extreme limit of very high densities could be a useful way to differentiate between the two cosmological scenarios. The different be-

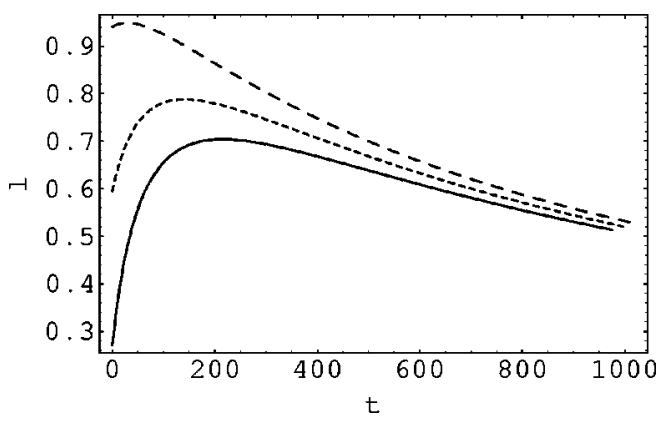

FIG. 11. Time variation of the absolute value $l$ of the ratio of the bulk viscous and thermodynamic pressures, $l=|\Pi / p|$ of the brane Universe with confined dissipative cosmological fluid with the coefficient of bulk viscosity proportional to the square root of the energy density $(s=1 / 2)$ for different numerical values of the integration constant $C_{1}: C_{1}=0.58$ (solid curve), $C_{1}=0.62$ (dotted curve), and $C_{1}=0.66$ (dashed curve) $\left(t_{0}=0\right)$. The constant $\tau_{0}$ has been normalized so that $\tau_{0}=1$. For all times for the chosen set of parameters the ratio of the pressures is smaller than 1 . 
havior of the energy density of cosmic matter ( $\rho \sim H$ in brane and $\rho \sim H^{2}$ in GR, respectively) leads, via the bulk viscous pressure evolution equation, to differences in the dynamics of the very early Universe, which perhaps can serve as a tool for testing the viability of brane model cosmology.

\section{ACKNOWLEDGMENTS}

The work of C.M.C. is supported by the Taiwan CosPA project and, in part, by the Center of Theoretical Physics at NTU and the National Center for Theoretical Science.
[1] L. Randall and R. Sundrum, Phys. Rev. Lett. 83, 3370 (1999).

[2] L. Randall and R. Sundrum, Phys. Rev. Lett. 83, 4690 (1999).

[3] P. Horava and E. Witten, Nucl. Phys. B460, 506 (1996).

[4] R. Maartens, "Geometry and dynamics of the brane-world," gr-qc/0101059.

[5] H.B. Kim and H.D. Kim, Phys. Rev. D 61, 064003 (2000).

[6] P. Binetruy, C. Deffayet, U. Ellwanger, and D. Langlois, Phys. Lett. B 477, 285 (2000).

[7] P. Binetruy, C. Deffayet, and D. Langlois, Nucl. Phys. B565, 269 (2000).

[8] H. Stoica, S.-H.H. Tye, and I. Wasserman, Phys. Lett. B 482, 205 (2000).

[9] D. Langlois, R. Maartens, and D. Wands, Phys. Lett. B 489, 259 (2000).

[10] H. Kodama, A. Ishibashi, and O. Seto, Phys. Rev. D 62, 064022 (2000).

[11] C. van de Bruck, M. Dorca, R.H. Brandenberger, and A. Lukas, Phys. Rev. D 62, 123515 (2000).

[12] C. Csaki, J. Erlich, T.J. Hollowood, and Y. Shirman, Nucl. Phys. B581, 309 (2000).

[13] R. Maartens, V. Sahni, and T.D. Saini, Phys. Rev. D 63, 063509 (2001).

[14] A.V. Toporenski, Class. Quantum Grav. 18, 2311 (2001).

[15] C.-M. Chen, T. Harko, and M.K. Mak, Phys. Rev. D 64, 044013 (2001).

[16] A.V. Frolov, Phys. Lett. B 514, 213 (2001).

[17] C. Csaki, M. Graesser, C. Kolda, and J. Terning, Phys. Lett. B 462, 34 (1999).

[18] J.M. Cline, C. Grojean, and G. Servant, Phys. Rev. Lett. 83, 4245 (1999).

[19] L. Anchordoqui, C. Nunez, and K. Olsen, J. High Energy Phys. 10, 050 (2000).

[20] T. Shiromizu, K. Maeda, and M. Sasaki, Phys. Rev. D 62, 024012 (2000).

[21] M. Sasaki, T. Shiromizu, and K. Maeda, Phys. Rev. D 62, 024008 (2000).

[22] K. Maeda and D. Wands, Phys. Rev. D 62, 124009 (2000).

[23] R. Maartens, Phys. Rev. D 62, 084023 (2000).
[24] A. Campos and C.F. Sopuerta, Phys. Rev. D 63, 104012 (2001).

[25] A. Campos and C. F. Sopuerta, Phys. Rev. D 64, 104011 (2001).

[26] C. Eckart, Phys. Rev. 58, 919 (1940).

[27] L.D. Landau and E.M. Lifshitz, Fluid Mechanics (Butterworth Heinemann, London, 1987).

[28] W. Israel, Ann. Phys. (N.Y.) 100, 310 (1976).

[29] W. Israel and J.M. Stewart, Phys. Lett. 58A, 213 (1976).

[30] W.A. Hiscock and L. Lindblom, Ann. Phys. (N.Y.) 151, 466 (1983).

[31] W.A. Hiscock and J. Salmonson, Phys. Rev. D 43, 3249 (1991).

[32] A.M. Anile, D. Pavon, and V. Romano, "The case for hyperbolic theories of dissipation in relativistic fluids," gr-qc/9810014.

[33] R. Maartens, Class. Quantum Grav. 12, 1455 (1995).

[34] R. Maartens, "Causal thermodynamics in relativity," astro-ph/9609119.

[35] L.P. Chimento and A.S. Jakubi, Class. Quantum Grav. 14, 1811 (1997).

[36] M.K. Mak and T. Harko, J. Math. Phys. 39, 5458 (1998).

[37] M.K. Mak and T. Harko, Gen. Relativ. Gravit. 30, 1171 (1998); 31, 273 (1999).

[38] L.P. Chimento, J. Math. Phys. 38, 2565 (1997).

[39] M.K. Mak and T. Harko, Aust. J. Phys. 52, 659 (1999).

[40] T. Harko and M.K. Mak, Int. J. Theor. Phys. 38, 1561 (1999).

[41] R. Maartens and J. Triginer, Phys. Rev. D 56, 4640 (1997).

[42] A.A. Coley and R.J. van den Hoogen, Class. Quantum Grav. 12, 1977 (1995).

[43] A.A. Coley and R.J. van den Hoogen, Phys. Rev. D 54, 1393 (1996).

[44] V.A. Belinskii, E.S. Nikomarov, and I.M. Khalatnikov, Sov. Phys. JETP 50, 213 (1979).

[45] V.A. Belinskii and I.M. Khalatnikov, Sov. Phys. JETP 42, 205 (1975).

[46] G.L. Murphy, Phys. Rev. D 8, 4231 (1973).

[47] W. Walter, Ordinary Differential Equations (Springer, New York, 1998). 